\title{
Meta
}

Journal des traducteurs

Translators' Journal

NIELSEN, SANDRO et SVEN TARP, dir. (2009) : Lexicography in

the 21st Century. In honour of Henning Bergenholtz.

Amsterdam/Philadelphia : John Benjamins, xi-341 p.

\section{Tanja Collet}

Volume 55, numéro 4, décembre 2010

De la localisation à la délocalisation - le facteur local en traduction

From Localization to Delocalization - The Local Factor in Translation

URI : https://id.erudit.org/iderudit/045695ar

DOI : https://doi.org/10.7202/045695ar

Aller au sommaire du numéro

Éditeur(s)

Les Presses de l'Université de Montréal

ISSN

0026-0452 (imprimé)

1492-1421 (numérique)

Découvrir la revue

Citer ce compte rendu

Collet, T. (2010). Compte rendu de [NIELSEN, SANDRo et SVEN TARP, dir. (2009): Lexicography in the 21st Century. In honour of Henning Bergenholtz.

Amsterdam/Philadelphia : John Benjamins, xi-341 p.] Meta, 55(4), 848-851.

https://doi.org/10.7202/045695ar 
faut en mesurer la perte, et, comme dans le cas du Canada, entre autres au Nouveau-Brunswick, l'accepter (p. 91). Comme dit Eco, qui prône la stratégie de la negoziazione (la négociation), s'agitil d'une souris ou d'un rat: mouse or rat? Après tout, ces deux animaux appartiennent à la même espèce: les rongeurs (Rodentia). Tout repose sur le choix que fera le traducteur entre une traduction orientée vers le texte d'arrivée ou celui de départ, d'où les enjeux de l'équivalence.

Le principe de l'équivalence, appliqué au droit, paraît relativement simple: on assume que, quel que soit le système juridique, des problèmes identiques se présentent partout qui appellent des solutions identiques, mais sont résolus par des moyens différents. Le hic? L'absence de contrepartie dans un autre système: Equity, trust, quasicontrat, quasi-délit. Voir aussi le contract anglais et sa célèbre consideration, notion inconnue en droit romano-germanique. L'ennui, nous dit le comparatiste Moréteau, "est que l'on ne trouve pas toujours une institution ou une technique équivalente» (p. 99).

Aussi l'équivalence fonctionnelle passe-t-elle, en droit comparé, pour la solution privilégiée en présence de systèmes comparables. Si l'équivalence fonctionnelle n'est pas la solution idéale, même entre systèmes proches (voir les critiques de Sarcevic, de Groot, p. 100), elle n'en constitue pas moins un pis-aller, un accommodement raisonnable que suivent les comparatistes depuis des décennies et qui, en l'absence d'une terminologie neutre (p. 108) ou d'une utopique ontologie (p. 112), est généralement préférable aux six autres procédés de traduction (calque, emprunt, traduction littérale, etc.), auxquels il est toujours possible de recourir selon les situations.

\section{L'apport de la pragmatique et de la traductologie}

Le cinquième chapitre est le plus théorique de l'ouvrage. Il s'adresse davantage au traductologue qu'au praticien en présentant un état des lieux, succinct mais complet, des théories contemporaines du $\mathrm{xx}^{\mathrm{e}}$ siècle. On part d'Austin et Searle avec les speech acts (p. 115), appliqués au langage du droit (p. 119), et on finit par les derniers développements de la traductologie, perçue comme transdiscipline. On y traverse de nombreux courants et croise nombre d'auteurs, depuis Jakobson, Nida, Holmes et Toury, jusqu'à... Derrida et son principe de déconstruction où la traduction est envisagée comme opérateur de différence (p. 127). Mais il conclut par la théorie - qu'il tient en haute estime - du skopos, ses sources et ses nombreux avatars, dont l'éthique selon Nord avec son principe de loyauté (p. 141), dans lequel Scarpa voit une version plus équilibrée de la dite théorie (p. 142). Le tout concourt à établir un cadre fonctionnaliste propre à répondre de façon optimale aux besoins de la traduction juridique.

En comparaison, le sixième et dernier chapitre fait figure d'enfant pauvre, avec ses cinq pages où l'auteur s'évertue à montrer la diversité des fonctions que peut revêtir la traduction juridique et qui se résume, en fait, au couple instrument / document. Il ne s'agit pas, non plus, d'une conclusion ou d'une esquisse de conclusion à l'ouvrage. Sans doute l'auteur n'en a-t-il pas ressenti la nécessité...

Ces quelques réserves n'enlèvent rien à la pertinence, à l'utilité et à l'intérêt de cet ouvrage. Existe-t-il d'ailleurs une méthode de traduction garantissant l'équivalence totale à recommander? À l'évidence, non, et en traduction juridique encore moins. La meilleure théorie de la traduction n'a jamais aidé, non plus, un traducteur à produire un meilleur texte, car on ne traduit pas à coups de théorie. Le terme théorie, en traduction, est par ailleurs surfait, voire déplacé, quand on compare la traductologie, science très sociale et très molle, aux sciences exactes. Les principes théoriques avancés par les traductologues, aussi savants soient-ils, ne viennent qu'en appoint, parallèlement à une pratique qui a fait ses preuves. L'analyse des mécanismes qui entrent en jeu dans l'opération traduisante révèle néanmoins les subtilités et les difficultés de la démarche du traducteur, elle ne le guide pas dans son épreuve. Or, chaque nouveau texte à traduire est un nouveau défi, un cas d'espèce requérant une stratégie particulière du traducteur, dont le savoir-faire reste encore la meilleure garantie de succès dans son entreprise. On peut toujours élever le débat jusqu'à la philosophie, rendre ainsi le traducteur plus intelligent, plus savant, ce qui n'est pas négligeable. C'est ce que l'auteur a cherché à faire, aussi, par cette présentation synthétique et forcément limitée des théories de la traduction juridique. Peut-être caressait-il l'espoir de voir le traducteur produire cette haute (alta) traduction que Sacco attribue au savoir du comparatiste (p. 25) et de parvenir à extraire la collaboration entre juristes et linguistes de son inexistence actuelle (p. 35). Seul l'avenir le dira.

Jean-Claude Gémar Université de Montréal, Montréal, Canada

Nielsen, Sandro et Sven Tarp, dir. (2009): Lexicography in the 21st Century. In honour of Henning Bergenholtz. Amsterdam/Philadelphia: John Benjamins, xi-341 p.

Lexicography in the 21st Century est le douzième volume à paraître dans la collection «Terminology and Lexicography Research and Practice (TLRP)» 
sous la direction de Marie-Claude L'Homme, de Kyo Kageura et de Juan C. Sager. Cette collection hyperspécialisée accueille, outre des monographies et des manuels, des travaux collectifs, nommés state-of-the-art volumes dans le descriptif de la TLRP parce qu'ils examinent les tout derniers développements dans les deux domaines visés par la série. Ce douzième volume se range dans cette troisième catégorie d'ouvrages. Ses deux directeurs, Sandro Nielsen et Sven Tarp, soulignent d'ailleurs dans leur introduction qu'il vise à présenter au lecteur «the state-of-the-art in lexicography at the beginning of the 21 st century » et qu'il cherche en outre à faire des suggestions « for future theoretical and practical work in the field" (p. x). Objectif ambitieux pour un travail collectif qui est, par ailleurs, le deuxième dans la série à prendre la forme d'un Festschrift. Dédié au lexicographe danois de renom Henning Bergenholtz, il se compose d'un ensemble d'articles - quatorze en tout - rédigés par des auteurs, qui ont dans bien des cas collaboré avec Bergenholtz à des projets dictionnairiques ou ont participé avec lui à l'élaboration de nouvelles approches théoriques et méthodologiques. C'est le cas notamment de Nielsen et de Tarp, mais aussi d'autres auteurs, tels que Wiegand, Svensén, Malmgren et Gouws, pour ne nommer que ceux-là. Les quatorze articles du volume sont précédés d'une introduction, qui esquisse la visée de l'ouvrage, et sont suivis d'une bibliographie, qui liste les publications principales de Bergenholtz de 1975 à 2009. Aux près de trois cents titres cités dans cette bibliographie, l'ouvrage fait suivre une liste des affiliations des auteurs et un index des notions-clés abordées dans les différents articles.

Le dédicataire du volume, Henning Bergenholtz, est à la fois un praticien et un théoricien accomplis. Il assume, depuis 1996, la direction du Centre de lexicographie de l'École des hautes études commerciales d'Aarhus au Danemark. Il est l'auteur ou le coauteur d'un nombre impressionnant de dictionnaires de langue danoise, allemande, et autres - la bibliographie en liste au moins vingt-quatre - se rangeant dans tous les genres confondus: monolingues et bilingues, généraux et spécialisés, imprimés et électroniques. Il est, en outre, l'un des fondateurs d'une nouvelle approche théorique qui a fait sa réputation en dehors des milieux germanophone et scandinave, à savoir la théorie des fonctions lexicographiques ou la lexicographie fonctionnelle. Parmi les autres fondateurs, il nous faut mentionner particulièrement Tarp, rattaché également au Centre de lexicographie de Aarhus. Dans ce compte rendu, il convient de consacrer quelques lignes à cette approche, développée à partir des années 1990, car elle constitue un des fils conducteurs de l'ouvrage.
En effet, à la page $x$ de l'introduction, on peut lire que les articles soumis par les différents auteurs visent à montrer «how the new theory of lexicographical functions can extend the forefront of the discipline by focusing on dictionary functions and how these can meet the needs of users in various types of user situations".

La lexicographie fonctionnelle de Bergenholtz et de ses collègues pose que la lexicographie constitue une discipline indépendante. Elle est ainsi en rupture avec la conception traditionnelle de la lexicographie qui tient cette discipline pour un sous-domaine de la linguistique, particulièrement appliquée.

[The modern theory of lexicographic functions] considers lexicography an independent scientific discipline and not, as in the case of a large number of lexicographers, to be a subdiscipline of linguistics. The subject field of lexicography is dictionaries, a human-made product, whereas the subject field of linguistics is language, i.e. something inherent in human beings. (Bergenholtz et Tarp 2003: 172)

La lexicographie fonctionnelle se distingue de ce fait radicalement «du triptyque "lexicographie, dictionnairique, métalexicographie" [...] mis en place, dans la tradition séparative quémadesque, par le Centre de recherche Métadif de Cergy-Pontoise», qui conçoit le premier membre du triptyque, donc la lexicographie, comme «une discipline de nature linguistique opérant sur des ensembles de mots », du moins selon Leroyer (2007: 4), également affilié au Centre de lexicographie d'Aarhus. Toujours selon ce même auteur, elle «se distingue aussi radicalement de l'approche phénoménologique de Wiegand» (Leroyer 2007: 4), bien qu'elle partage avec cette approche, comme le soulignent Bergenholtz et Tarp (2003), le postulat que les dictionnaires ne sont pas des répertoires d'items lexicaux mais bien plutôt des outils façonnés par des êtres humains pour résoudre des problèmes spécifiques. Étant donné ce postulat, la lexicographie fonctionnelle pose que la responsabilité première du lexicographe est d'articuler toute réflexion théorique ou méthodologique autour de trois axes complémentaires: les utilisateurs de dictionnaires, les situations spécifiques dans lesquelles ils œuvrent et les difficultés qu'ils peuvent y rencontrer. Elle identifie au moins deux types de situations ${ }^{1}$, les unes axées sur la communication et les autres sur la cognition, dans lesquelles les utilisateurs peuvent se heurter à des problèmes particuliers qui les pousseront à consulter un dictionnaire dans l'espoir d'y trouver une solution. Dans ce contexte, les tenants de l'approche appellent fonction lexicographique la capacité d'un dictionnaire de répondre aux besoins, par exemple communicatifs ou cognitifs, ressentis 
par un groupe d'utilisateurs aux caractéristiques précises et agissant dans une situation particulière.

A lexicographic function of a given dictionary is to provide assistance to a specific user group with specific characteristics in order to cover the complex of needs that arise in a specific type of user situation. (Bergenholtz et Tarp 2003: 176)

La lexicographie fonctionnelle distingue, par conséquent, au moins deux types de fonctions: des fonctions communicatives et des fonctions cognitives. Les premières sont «associées aux activités de production, de traduction, de réception ou de correction de texte» (Leroyer 2007: 6). Les deuxièmes, quant à elles, sont "associées à un désir [de la part de l'utilisateur] d'acquérir ou de vérifier des connaissances par le biais d'une consultation sporadique ou systématique, en situation d'apprentissage d'une langue ou d'un domaine de spécialité» (Leroyer 2007: 6). Un dictionnaire peut être mono- ou multifonctionnel, selon les tenants de l'approche. Cependant, il ne peut contenir que des données lexicographiques pertinentes et doit recourir à des structures de présentation qui lui permettront de réaliser sa ou ses fonctions.

Étant donné la visée de l'ouvrage, plusieurs contributions adoptent la perspective fonctionnelle de Bergenholtz et de ses collègues. C'est le cas notamment de celles de Gouws, de Nielsen, de Tarp, de Malmgren, de Leroyer et de FuertesOlivera et, dans une certaine mesure, également de celles de Geeb et de Herbst. Aucune de ces contributions ne cherche à enrichir l'approche, mais s'en inspire bien plutôt pour résoudre des problèmes de type méthodologique. Elles se trouvent réparties sur les cinq sections de l'ouvrage qui abordent chacune des thématiques différentes. Celles-ci vont de questions reliées aux structures, aux fonctions et aux utilisateurs de dictionnaires à d'autres encore reliées à la lexicographie spécialisée, à la lexicographie électronique et au traitement dictionnairique de la phraséologie. C'est ainsi que ces contributions illustrent bien l'étendue de cette approche, qui s'applique sans problème tant dans le domaine traditionnel du dictionnaire papier que dans celui plus récent du dictionnaire virtuel et ce, quel que soit le type, général ou spécialisé, ou le nombre de langues traitées.

Gouws (p. 3-21) et Tarp (p. 43-62) se penchent dans leurs contributions respectives sur des problèmes structuraux et d'accès posés, entre autres, par l'inclusion d'items lexicaux complexes (des mots composés et dérivés, par exemple, mais aussi des items de type phraséologique). S’inspirant des possibilités offertes par les nouvelles technologies, Tarp en vient à envisager un dictionnaire électronique composé d'articles dynamiques (dynamic article, p. 57) dont le contenu et la structure varient selon les paramètres actionnés par l'utilisateur lors de sa recherche. Il conclut son texte en signalant qu'une nouvelle ère a commencé en lexicographie, celle d'une individualisation accrue du produit lexicographique:

Nobody knows how dictionaries will develop in the future, but one direction will probably be the 'individualization' of the lexicographic product, adapting it to the concrete needs of a concrete user in a concrete situation and providing much quicker and easier access to the relevant data. (p. 61)

Nielsen (p. 23-41), pour sa part, s'occupe à définir des paramètres de type fonctionnel pour l'évaluation et la comparaison de dictionnaires. Il déplore que ce type d'exercice se fasse trop souvent dans une perspective linguistique.

A study of various theoretical contributions to dictionary criticism, [...], reveal [sic] a relatively strong bias towards a linguistic approach to dictionary reviewing. They generally treat dictionaries as containers of purely linguistic concepts and focus on linguistic categories such as word classes, [...] grammatical information [...], semantic and encyclopaedic information and equivalents. This is in line with the traditional understanding of dictionaries [...]. (p. 26-27)

Il espère que sa méthode, qui respecte l'indépendance disciplinaire de la lexicographie et qui considère le dictionnaire comme une entité tripartite constituée d'un contenu, d'une forme et d'une ou plusieurs fonctions, mènera à des évaluations davantage informatives.

The above proposed basis for a framework [...] may result in reviews that actually contribute to the development of lexicographic theories and dictionary compilation. (p. 38)

Dans son article, Malmgren (p. 93-102) s'intéresse à une sous-fonction de la fonction communicative, à savoir celle qui concerne la production de textes. Il examine plus particulièrement comment des données axées sur la production, telles que par exemple des renseignements reliés à l'usage, à la synonymie et à la cooccurrence, sont incorporées dans le plus important dictionnaire du suédois, le Svensk ordbok. Quant à Leroyer (p. 103-122), il se sert de la lexicographie fonctionnelle pour montrer comment cette approche peut mener à une transformation de fond en comble d'un genre lexicographique peu étudié: le dictionnaire pour touristes. Fuertes-Olivera (p. 161-178), pour sa part, se place dans une perspective fonctionnelle pour concevoir une composante importante de tout dictionnaire spécialisé: l'introduction systématique. 
Systematic introductions are separate dictionary components, placed either in the front matter or in the back matter [...], and aim at providing help in cognition-oriented and communication-oriented user situations. (p. 162)

Il fait ses réflexions dans le contexte d'un projet de lexicographie spécialisée multilingue (danois, anglais, espagnol) dans le domaine de la comptabilité et émanant du Centre de lexicographie d'Aarhus. Geeb (p. 207-234) se base sur les travaux en lexicographie fonctionnelle dans sa description de lookedup4you, un robot virtuel de type chatbot qui se constitue d'une composante renfermant des données lexicographiques. Herbst (p. 281-308), enfin, s'intéresse aux dictionnaires pour apprenants. Il se penche sur le traitement dans ces dictionnaires de phénomènes qui peuvent faire obstacle en situation de production. Il s'agit particulièrement de phénomènes qui mettent en cause l'axe syntagmatique de la langue, comme la complémentation du verbe mais aussi et surtout la cooccurrence.

Les six contributions restantes font le bilan de recherches sur des sujets chers au dédicataire mais sans adopter pour autant une perspective fonctionnelle. Elles s'inspirent plutôt d'autres travaux de Bergenholtz effectués dans des domaines aussi variés que l'aménagement linguistique, la lexicographie spécialisée, la lexicographie électronique et la lexicographie générale. Ainsi, Vikør (p. 123-143) commence son texte par un examen, plutôt critique, d'un article publié en 2006 par Bergenholtz et Gouws: «How to Do Language Policy with Dictionaries ? ${ }^{2}$ " Il enchaîne avec une comparaison de plusieurs dictionnaires d'orthographe utilisés dans le monde germanique, particulièrement les pays scandinaves et les Pays-Bas. Svensén et L'Homme, pour leur part, citent tous les deux le manuel de lexicographie spécialisée publié par Bergenholtz et Tarp ${ }^{3}$ en 1995. Svensén (p. 147-159) commente sur la révision en profondeur de l'arbre de domaine conçu pour un dictionnaire spécialisé en lexicographie: le Nordisk leksikografisk ordbok. L'Homme (p. 237-256), quant à elle, décrit la méthodologie retenue pour l'inclusion de collocations dans le DiCoInfo, une base de données terminologique de langue française. Cette méthodologie ne se base pas, cependant, sur les travaux de Bergenholtz, mais sur la lexicologie explicative et combinatoire développée par un autre lexicographe de renom, à savoir Igor Mel'čuk ${ }^{4}$. Quant aux contributions de Prinsloo et de Jónsson, elles se situent dans le sous-domaine de la lexicographie électronique. Prinsloo (p. 181-206) examine la place centrale que le corpus occupe dans la lexicographie du $\mathrm{XxI}^{\mathrm{e}}$ siècle. Jónsson (p. 257-280), pour sa part, décrit en détail un projet lexicographique d'envergure, la construction d'un wordnet islandais. Wiegand (p. 63-89), finalement, s'intéresse à la structure de l'article dictionnairique. Cependant, on peut regretter que cet article, traduit de l'allemand par Gouws, soit très dense et, de ce fait, difficile à lire pour tout lecteur à qui les travaux de cet auteur ne sont pas familiers.

En conclusion, on peut dire que ce Festschrift est bien conçu. En effet, il rend, comme il se le doit, non seulement hommage à Bergenholtz, mais se compose de contributions, qui sont toutes sans exception d'une excellente qualité et illustrent d'une façon convaincante l'importance pour la lexicographie du $\mathrm{xxI}^{\mathrm{e}}$ siècle des innovations, tant théoriques que pratiques, apportées par Bergenholtz au cours de sa longue et très riche carrière. Par le biais de leurs contributions, les auteurs célèbrent de plus l'impact à différents degrés que Bergenholtz a eu dans leur vie professionnelle. Ils invitent ainsi le lecteur à faire comme eux, qui «have taken off from their own experiences and research journeys in the light of Henning Bergenholtz's rich work, and gone in many of their own exciting directions» (p. x).

Tanja Collet University of Windsor, Windsor, Canada

\section{NOTES}

1. Dans sa contribution, à la page 47 de l'ouvrage, Tarp liste non pas deux mais quatre situations: des situations communicatives, cognitives, opératives et interprétatives. Il ajoute de plus que la liste est loin d'être close. Cependant, les situations communicatives et cognitives correspondent aux situations de base retenues par l'approche fonctionnelle dans sa version classique.

2. Bergenholtz, H. et Gouws, R. H. (2006): How to Do Language Policy with Dictionaries?. Lexikos. 16: 13-45.

3. Bergenholtz, H. et TARP, S., dir. (1995): Manual of Specialised Lexicography. The Preparation of Specialised Dictionaries. Amsterdam/Philadelphia: John Benjamins.

4. Mel'Čuk, I. et al. (1984-1999): Dictionnaire explicatif et combinatoire du français contemporain. Montréal: Presses de l'Université de Montréal.

\section{RÉFÉRENCES}

Bergenholtz, Henning et Tarp, Sven (2003) : Two opposing theories: On $\mathrm{H}$. E. Wiegand's recent discovery of lexicographic functions. Hermes, Journal of Linguistics. 31:171-196.

Leroyer, Patrick (2007): Terminologie et dictionnaires: la porte des utilisateurs. Communication faite lors du colloque Terminologie: approches transdisciplinaires tenu à l'Université du Québec en Outaouais. Consultée le 8 mars 2010, <www.uqo.ca/terminologie2007/documents/Leroyer.pdf $>$. 